\title{
Post-cranioplasty Blindness
}

\author{
Eunhye Lee, Inkyeoung Kim \\ Departments of Neurosurgery, Kangwon National University School of Medicine, Chuncheon, Korea
}

Acute visual loss resulting from an uncomplicated cranioplasty is rare. A 52-year-old man presented with computed tomography (CT)-proven acute subdural hematoma in the right frontotemporoparietal area. The patients got a standard craniotomy in supine position. But on postoperative day 24 , he was diagnosed postoperative epidural and subdural empyema. The patient underwent infected bone flap removal and evacuation of epidural and subdural empyema. Four months later, he got right frontotemporoparietal cranioplasty with artificial bone (Medpor) after controlling of infection. But on postoperative day 15 , he complained of inability to see with his right eye. Brain and orbital CT, magnetic resonance imaging scan performed postoperatively did not show any new intracranial or intraorbital pathology. There were not any adverse events such as excessive pressure placed on the eyeball or bulky retraction of frontal skin flap. Visual evoked potential revealed prechiasmatic lesion. Fundoscopy showed slightly pallor optic disc of right side. The patient's vision in the right eye remained no light perception permanently. The reason and pathophysiology of the mechanism of this complication should be discussed through reporting more cases.

Key Words: Brain $\cdot$ Craniotomy $\cdot$ Empyema, subdural $\cdot$ Evoked potentials, visual $\cdot$ Eye $\cdot$ Humans $\cdot$ Magnetic resonance imaging $\cdot$ Male $\cdot$ Pallor $\cdot$ Polyethylenes $\cdot$ Subdural space $\cdot$ Supine position $\cdot$ Tomography, X-ray computed $\cdot$ Medpor

\section{INTRODUCTION}

Blindness resulting from a cranioplasty is uncommon. The author report a rare case of acute ipsilateral blindness that occurred after a standard frontotemporoparietal cranioplasty using artificial bone (Medpor) performed in supine position resulted in permanent visual loss in the ipsilateral eye.

\section{CASE REPORT}

A 52-year-old man presented with computed tomography (CT)-proven acute subdural hematoma in the right frontotemporoparietal area (Fig. 1A). The patients got a standard craniotomy in supine position. The patient woke up from surgery and fully alert without any neurological deficit. But on postoperative day 24, he had scalp swelling and periorbital swelling with high fever of $39^{\circ} \mathrm{C}$. Brain CT and magnetic resonance imaging (MRI) scan were done and he was diagnosed postoperative epidural and subdural empyema. The patient underwent infected bone flap removal and evacuation of epidural

Corresponding author: Inkyeong Kim

Department of Neurosurgery, Kangwon National University Hospital, Baengnyeong-ro 156, Chuncheon 24289, Korea

Tel: +82-33-258-9204, Fax: +82-33-258-9489

E-mail: deargrape@naver.com
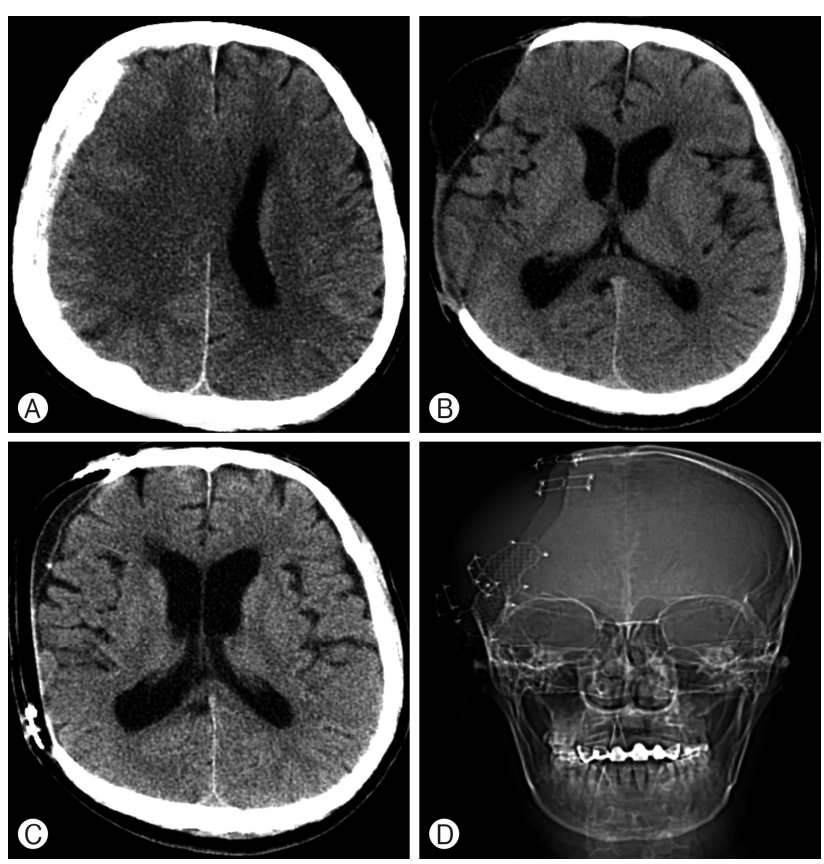

Fig. 1. (A) Initial brain computed tomography (CT) scan showed acute subdural hematoma on the right frontotemporoparietal lobe. (B) Brain CT after 7 months. It revealed skull defect of right hemisphere with mild scalp bulging with cerebrospinal fluid collection. (C) Immediate post-cranioplasty brain CT showed that right frontotemporoparietal cranioplasty were performed with artificial bone (Medpor). (D) Immediate post-cranioplasty skull X-ray. 

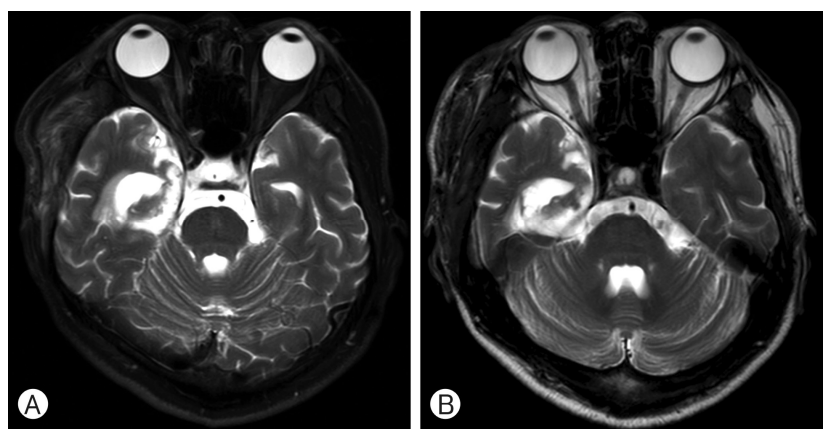

Fig. 4. Pre and post-cranioplasty T2-weighted magnetic resonance image (MRI). (A) Pre-cranioplasty T2-weighted MRI showed mild kinking of right optic nerve in the intraorbital cavity. (B) Postcranioplasty T2-weighted MRI showed slightly aggravated kinking of right optic nerve.

and subdural empyema. The pathogen was proven Staphylococcus aureus (methicillin-resistant Staphylococcus aureus [MRSA]). He got 6 weeks of IV antibiotics therapy of cefazolin

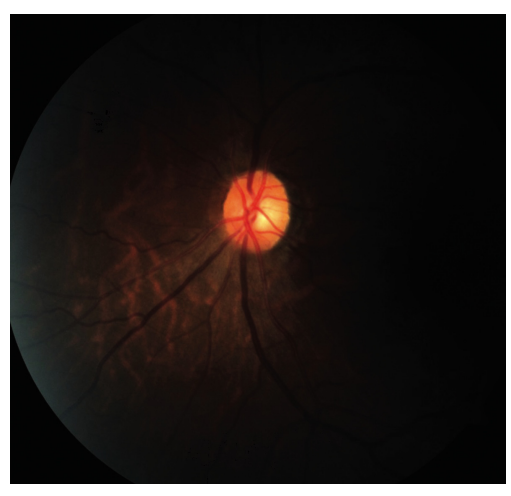

Fig. 2. Post-cranioplasty fundus photo with pale optic nerve of right side.
$2 \mathrm{~g}$ q8h after consultation with the infectiology medicine.

Four months later, the patient was considered of complete control of infection. He got right frontotemporoparietal cranioplasty with artificial bone (Medpor) (Fig. 1C, D). Intraoperatively, we did not open the dura and did not manipulation of brain parenchyme and the optic nerve. And there was no external compression of periorbital area with minimal scalp retraction during the cranioplasty. Immediate postoperatively, the patient was fully recovered without any neurological deficit. But on postoperative day 15, he complained of blurred vision with his right eye. External examination of the right eye was unremarkable except mild palpebral swelling that is possible postoperatively. On ophthalmologic examination, the patient revealed no perception of light and afferent pupillary defect in the right eye. The pupil size of affected eye was $3 \mathrm{~mm}$ with fixed. Extraocular movement was normal. Fundoscopy showed slightly pallor optic disc of right side (Fig. 2). Visual evoked potential revealed prechiasmatic lesion (Fig. 3). But brain and orbital CT, MRI scan performed postoperatively did not show any new intracranial or intraorbital pathology. Only remarkable thing on MRI image was that kingking of the course of the right optic nerve and asymmetrical prominency of the right ophthalmic artery compared to left side, pre- and post-operatively (Fig. 4). Although the patient made an excellent neurological recovery, vision in the right eye remained no light perception permanently.

\section{DISCUSSION}

The pathophysiology of this event is unclear. It is mainly
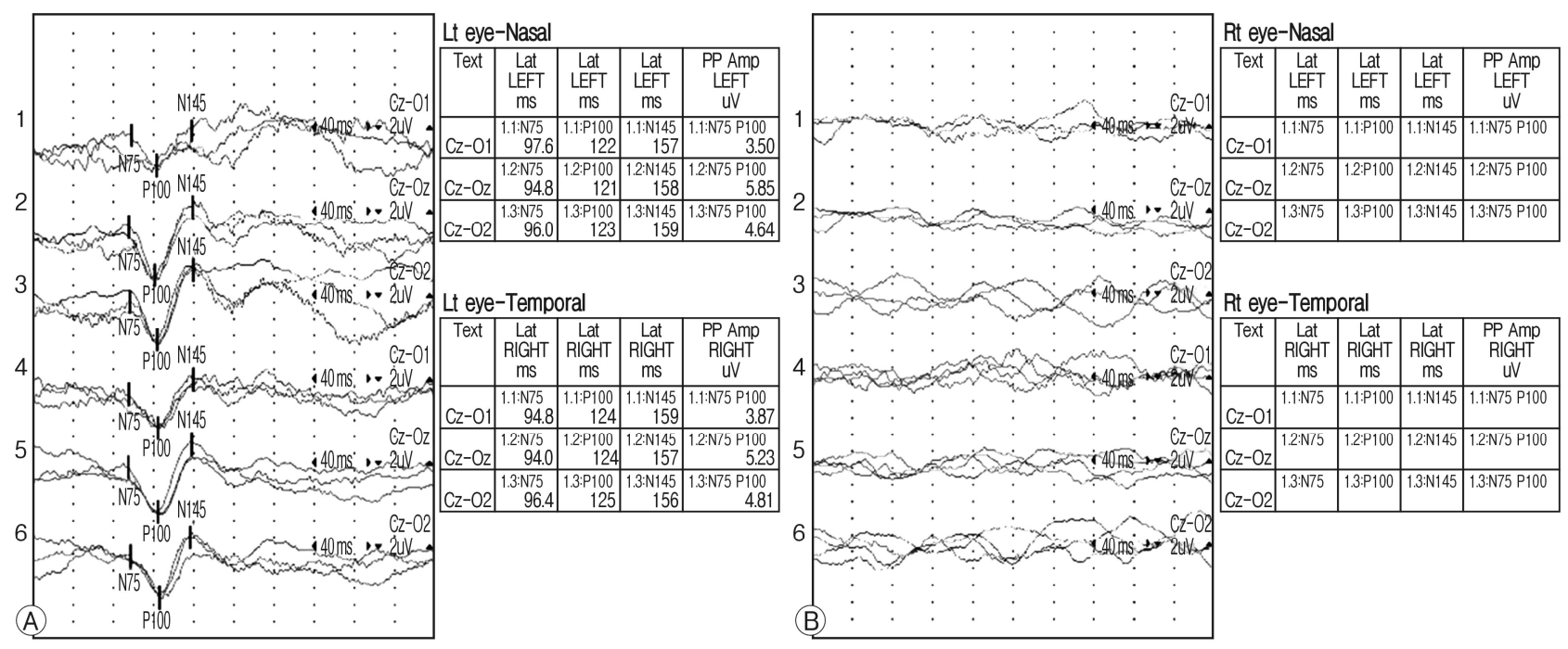

Fig. 3. Visual evoked potential after cranioplasty. (A) Left visual evoked potential (VEP) shows normal wave and amplitude. (B) Right VEP showed no recordable VEP in the whole field that revealed prechiasmatic lesion. Lt: left; Rt: right. 
caused by intraoperative nerve injury. In our patients, we could exclude possible etiological factors such as abnormal systemic and ocular conditions, causing ischemia in intra-or- bital structures, intra-operative hypotension, carotid atherosclerosis, and ocular vasospasm etc. ${ }^{2)}$. Accordingly we speculate that the complication seen in our case was most likely related to intraorbital ischemia initiated by a collapse of the arterial and venous channels in the orbit and/or to direct or indirect contusion on the intra-orbital structures ${ }^{2}$. This situation could be produced by inadvent pressure placed on the eyeball with a bulky retracted frontal skin flap, but not corresponding our patient ${ }^{2}$. Similar reports exist on major opera- tion such as an. Clipping or brain tumor surgery ${ }^{1,2,5}$. If they occurred, the condition of the patients were irreversible. The degree of visual recovery seems to be dependent of the duration and severity of retinal ischemia by orbital compression ${ }^{2}$. There is another possible hypothesis that postulating that the cerebrospinal fluid (CSF) circulates in three subarachnoidal compartment: the intracranial area; and the intraorbital subarachnoidal spaces of each optic nerve. In this condition, it appears that the subarachnoid spaces of each optic nerve become isolated from the cerebrospinal dynamics of the intracranial space by scar tissue ${ }^{3)}$. It might to be similar mechanism of neurologic deterioration with sinking skin flap syndrome, caused by negative gradient between atmospheric and intracranial pressure, which is aggravated by change in the CSF compartment following CSF hypovolemia after craniectomy ${ }^{4}$. Of course, the patient of our report may not have absolutely same mechanism with the cases of post-decompression visual loss.

After all, a drop in systemic blood pressure reducing optic nerve blood flow already compromised by the previous insult to optic nerve could be possible cause ${ }^{1)}$. Unfortunately there is no satisfactory treatment ${ }^{2}$. Also, preventive or restorative interventions are unclear ${ }^{5}$. Possible risk factors are pre-operative decreased visual acuity or visual field, papilledema or optic atrophy. A better understanding of this disorder would be obtained by the complete preoperative ophthalmic examination on patients who do develop visual loss following cranioplasty ${ }^{1)}$. This complication should be discussed with highrisk patients and their families preoperatively ${ }^{1}$. If patients at high risk could be identified prior to surgery, performing intraoperative visual evoked potential testing and continuous blood pressure monitoring would aid greatly in understanding the mechanism of visual loss ${ }^{11}$.

\section{CONCLUSION}

We describe the rare case of post-cranioplasty optic neuropathy and sudden visual loss after an uncomplicated cranioplasty in the supine position of an acute subdural hematoma patient. The reason and pathophysiology of the mechanism of this complication should be discussed through reporting more cases.

\section{REFERENCES}

1. Beck RW, Greenberg HS: Post-decompression optic neuropathy. J Neurosurg 63:196-199, 1985

2. Kang S, Yang Y, Kim T, Kim J: Sudden unilateral blindness after intracranial aneurysm surgery. Acta Neurochir (Wien) 139:221226, 1997

3. Killer HE, Jaggi GP, Flammer J, Miller NR, Huber AR, Mironov A: Cerebrospinal fluid dynamics between the intracranial and the subarachnoid space of the optic nerve. Is it always bidirectional? Brain 130:514-520, 2007

4. Kim SY, Lee CH, Park IS, Hwang SH, Han JW: Sinking skin flap syndrome after craniectomy in a patient who previously underwent ventriculoperitoneal shunt. Korean J Neurotrauma 8:149152, 2012

5. Vahedi P, Meshkini A, Mohajernezhadfard Z, Tubbs RS: Postcraniotomy blindness in the supine position: Unlikely or ignored? Asian J Neurosurg 8:36-41, 2013 